\title{
Inter-individual differences in fMRI entropy measurements in old age
}

\author{
Moses O. Sokunbi, Graduate Student Member, IEEE, Roger T. Staff*, Gordon D. Waiter, Trevor S. \\ Ahearn, Helen C. Fox, Ian J. Deary, John M. Starr, Lawrence J. Whalley, and Alison D. Murray
}

\begin{abstract}
We investigated the association between individual differences in cognitive performance in old age and the approximate entropy (ApEn) measured from functional magnetic resonance imaging ( $\mathrm{FMRI}$ ) data acquired from 40 participants of the Aberdeen Birth Cohort 1936 (ABC1936) while undergoing a visual information processing task: inspection time (IT). Participants took a version of the Moray House Test No. 12 (MHT) at age 11, a valid measure of childhood intelligence. The same individuals completed a test of non-verbal reasoning (Raven's Standard Progressive Matrices [RPM]) aged about 68 years. The IT, MHT and RPM scores were used as indicators of cognitive performance. Our results show that higher regional signal entropy is associated with better cognitive performance. This finding was independent of ability in childhood but not independent of current cognitive ability. ApEn is used for the first time to indentify a potential source of individual differences in cognitive ability using fMRI data.
\end{abstract}

Index Terms-Approximate Entropy (ApEn), BOLD, fMRI, Inspection time, Raven's matrices and Scottish Mental Survey.

\section{INTRODUCTION}

$\mathrm{T}$ HE origins of inter-individual differences in cognitive ageing are unclear. Cognitive performance can differ significantly between individuals of similar age and agerelated pathology. Identifying the structural and functional characteristics that accompany successful ageing may; 1) facilitate interventions that would promote the acquisition of

Manuscript received February 14, 2011; revised May 31, 2011. This work was supported in part by the Alzheimer Research Trust, in part by the Wellcome Trust, and in part by a SINAPSE (www.sinapse.ac.uk) studentship. Asterisk indicates corresponding author.

M.O. Sokunbi, G.D. Waiter, T.S. Ahearn and A.D. Murray are with the Aberdeen Biomedical Imaging Centre, SINAPSE Collaboration, University of Aberdeen, Foresterhill, Aberdeen, Scotland, United kingdom.

*R.T. Staff is with the Department of Nuclear Medicine, Aberdeen Royal Infirmary, NHS-Grampian, Foresterhill, Aberdeen, Scotland, United Kingdom (phone:+44(0)1224-552239; fax: +44(0)1224-554753;

email: r.staff@abdn.ac.uk).

J.M. Starr is with Geriatric Medicine unit, University of Edinburgh, Edinburgh, Scotland, United Kingdom.

H.C. Fox and L.J. Whalley are with the University of Aberdeen, Aberdeen, Scotland, United Kingdom.

I.J. Deary is with the Centre for Cognitive Ageing and Cognitive Epidemiology, University of Edinburgh, Edinburgh, Scotland, United Kingdom. such characteristics; 2) identify individuals at risk of future cognitive decline; 3 ) identify a target for potential therapies that would maintain such characteristics.

Brayne et al. [1] identified education as enabling individuals to delay the effects of dementia pathology on cognition whilst not preventing such pathology itself; therefore, education may be considered as a proxy for cognitive reserve. Cognitive reserve is a phrase used to explain the discrepancy between pathology attributed to ageing and disease and the degree of cognitive impairment exhibited in an individual. There is however no biological marker [2]-[4] for cognitive reserve, in addition there is no obvious mechanism through which its proxies are implemented. An alternative perspective on this phenomenon is to consider reserve as the 'adaptive capacity' of the system. Lipsitz [5] has argued that greater underlying system complexity better enables a system to restore the steady-state after perturbation and therefore, complexity may be a measure of adaptive capacity.

Complexity refers to the difficulties arising when describing or predicting a signal. Lipsitz [6] stated that, with ageing and disease, there is a loss of complexity in the dynamics of many integrated physiological processes. Normal physiological function requires the integration of intricate networks of control systems, feedback loops, and other regulatory mechanisms to enable an organism simultaneously to perform the many necessary and varied activities.

There are indications that measuring the complexity of the output signals of the brain might be a useful marker of ageing and disease-related decline. Researchers have argued that there is a general loss of complexity with ageing and disease [7],[5],[8]. Chaotic and complex behaviours indicate a healthy system whereas more predictable behaviours would be linked to pathological states [9].

Entropy is a concept used to quantify complexity. Increased uncertainty and complexity is quantified in higher entropy, while reduced uncertainty and complexity is quantified in lower entropy. Lipsitz defined ageing as a loss of "entropy and fractality" [6]. Also, a loss of entropy in cellular processes is observed during ageing [10] which implies a progressive reduction in an individual's functional reserve [11]. Approximate Entropy (ApEn) is a measure of the complexity of a system. A high ApEn indicates unpredictability and random variation (high complexity), whereas low ApEn indicates predictability and structure (low complexity) [12]. ApEn is applicable to a variety of systems such as stochastic, deterministic and composite systems.

Copyright (C 2011 IEEE. Personal use of this material is permitted. However, permission to use this material for any other purposes must be obtained from the IEEE by sending an email to pubs-permissions@ieee.org 
ApEn may correlate with "latent" or subclinical changes often undetected by other more classical time series analysis. It provides effective discriminatory capability in instances where measures such as spectral and autocorrelation analyses exhibit minimal distinctions [13]. ApEn changes have often been seen to be predictive of subsequent clinical changes. This has facilitated its application to numerous areas. Within medicine and biology, it has been applied to studies of hormone fluctuations. Using plasma concentration time series ApEn identified subtle system changes and insights separate from those given by pulse detection algorithm [14]. It has been used to identify gender and age-related differences in heart rate dynamics [15] and been used to classify arrhythmias [16]. It has also been used to study the human respiratory movement [17] showing that respiratory movement and electroencephalogram (EEG) signals are more regular during stage IV sleep than during other stages of consciousness. The relationship of hypothermia and EEG signals has been studied using ApEn [18] showing that the ApEn of the EEG changed in a sigmoidal fashion during cooling and rewarming. The relationship of the ApEn of EEG signals with Alzheimer's disease has been investigated [19] showing that the degree of complexity of EEGs from control subjects is higher.

In order to assess the relationship between entropy and cognition in the context of ageing, we investigated its relationship with a cognitive task that changes with age. Recent attempts to explain cognitive ageing have focused on a small number of more "fundamental cognitive mechanisms" [20]. One hypothesis in cognitive ageing is that speed of information processing has a special place in understanding what happens in the older person's brain [21],[22]. This hypothesis suggests that processing speed might not simply be one of many domains of cognitive function that decline with age, but that processing speed may be a phenomenon that underpins other functions. That is, if speed of information processing slows down, then this becomes the rate limiting step for other important cognitive functions. One technique for measuring processing speed is to use an inspection time task. In this study we employ a psychophysical task of speed of information processing that assesses the efficiency of iconic memory in the early stages of visual information processing. Inspection time (IT) is a visual backward masking task [23]. The task requires only a very simple visual discrimination, one that is almost error-free at longer stimulus exposure durations, such as the $150 \mathrm{~ms}$ duration included in the present study.

In the absence of dementia, the strongest predictor of cognitive function in old age is cognitive function in youth, accounting for around half of the variance [24]. Investigators have stressed that in addition to childhood intelligence there are many relevant environmental influences such as education, occupational complexity, a socially-engaged and intellectually stimulating lifestyle and good nutrition [25]. Along with unknown genetic factors, these are likely contributors to late life abilities in addition to education [4]. It is unclear if differences in adaptive capacity are related to current cognitive abilities or pre-morbid abilities. A schematic representation of how these influences may fit together is shown in Fig. 1. We hypothesize that there is an association between the entropy of the measured fMRI BOLD signal and the ability to perform an information possessing task (IT). The figure also shows a simple model for lifelong cognitive ageing. In this model we hypothesize that childhood ability has a direct effect on late life abilities and an indirect effect mediated by life experience brought about by the factors described above. The figure also indicates that these lifelong measures either separately or in combination influence our fMRI entropy measure and processing speed. We are in a privileged position to examine, in older people, whether estimates of entropy are explained by current or prior (childhood) intelligence.

The aim of this study is to investigate, in an age homogeneous sample of older people, differences in cognitive performance and information processing speed and their associations with differences in the ApEn calculated from fMRI data acquired during performance of the inspection time task. We also investigate whether these associations are related to childhood or current intelligence. We hypothesize that higher levels of ApEn (higher complexity) will be associated with better cognitive performance.

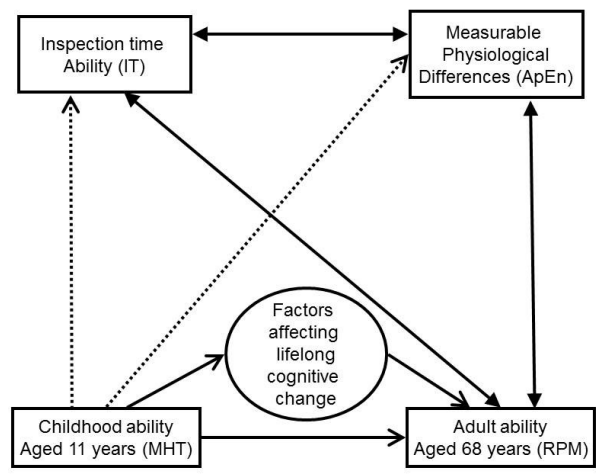

Fig.1. A schematic representation of how cognitive abilities measured over the life span influence the hypothesized association between IT abilities and entropy. Single headed arrows indicate potential causal influences and double headed arrows represent potential correlations. The dotted lines represent association where we found no significance.

\section{MATERIALS AND METHODS}

\section{A. Participants}

All were surviving participants of the 1947 Scottish Mental Survey (1947SMS) [26] when about 95\% eligible children born in 1936 and attending a Scottish school on $4^{\text {th }}$ June 1947 sat a group administered IQ-type test, a version of the Moray House Test No. 12 (MHT). About age 64, survivors were recruited to a longitudinal study of health and cognitive ageing, the Aberdeen Birth Cohort 1936 (ABC1936). From the ABC1936 sample, 58 individuals aged 68 - 70 (28 female) with an age $11 \mathrm{IQ}$, calculated from the Moray House Test (MHT) raw scores, between 85 and 115 (i.e. within 1 Standard Deviation (SD) of the sample mean) were invited for fMRI scanning. The age 11 IQ score was calculated by deriving a standard IQ-type scale within a mean of 100 and SD of 15 from the ABC1936 sample's age 11 MHT raw scores using the whole $\mathrm{ABC} 1936$ recruited at age $64(\mathrm{~N}=661)$. Hence, only

Copyright (C) 2011 IEEE. Personal use of this material is permitted. However, permission to use this material for any other purposes must be obtained from the IEEE by sending an email to pubs-permissions@ieee.org 
participants with IQs greater than 84 and less than 116 were considered for this study.

The study was approved by the Grampian Research Ethics Committee. Written informed consent was obtained by a trained research nurse.

\section{B. Indicators of Cognitive Performance}

\section{Inspection Time Testing}

Inspection Time (IT) tasks used in the imaging sessions followed closely those described by [27] and detailed in [28, 29]. Participants were instructed to make a simple visual discrimination i.e. to indicate which of two parallel, vertical lines of markedly different lengths, was longer (Fig. 2.).

58 participants volunteered for the functional imaging study and all had previously successfully completed the IT task as part of their cognitive testing. Immediately before brain imaging, participants practised the task to ensure their complete familiarity with the IT task demands. They were instructed that accuracy and not speed of responses was being assessed.

The IT experiment took place in the MRI scanner. We have previously described the brain-imaging inspection time session and procedures, and we reported on the functional anatomy associated with performing the IT task, and the extent to which the BOLD response mediates the association between inspection time performance and intelligence [28, 29]. Briefly, in the imaging IT session, twenty trials were presented at each of eight durations: $6,12,25,37,50,75,100$, and $150 \mathrm{~ms}$. Paradigms were programmed in Presentation (Neurobehavioral Systems Inc., CA) with instructions and stimuli presented visually on a computer monitor and viewed via a mirror on the head coil. The eye-to-screen distance was about $5 \mathrm{~m}$. Visual acuity was assessed immediately before scanning and corrected with MRI compatible lenses as necessary. Pushbutton units were provided to allow participants to log responses. Participants indicated the position of the longer line by pressing a key with the left index finger (for 'left') or a key with the right index finger (for 'right'). The same optimal inter stimulus interval (ISI) sequence was used for all trials. The same random sequence of stimulus durations was presented to all subjects.

\section{Cognitive tests}

All participants had taken part in 1947SMS aged 11, when they sat a version of the Moray House Test (MHT) No. 12 of general mental ability (intelligence). MHT is a group administered test with a time limit of $45 \mathrm{~min}$, a maximum score of 76 and a range of questions including verbal, numerical and spatial reasoning. In a national subset of 1000 children, scores on MHT correlated about 0.8 with the individually administered Stanford-Binet IQ test [26].

In order to test current cognitive ability, participants completed an individually administered test of non-verbal reasoning (Raven's Standard Progressive Matrices [RPM]) [30] at age 68, not greater than 8 months prior to fMRI acquisition.

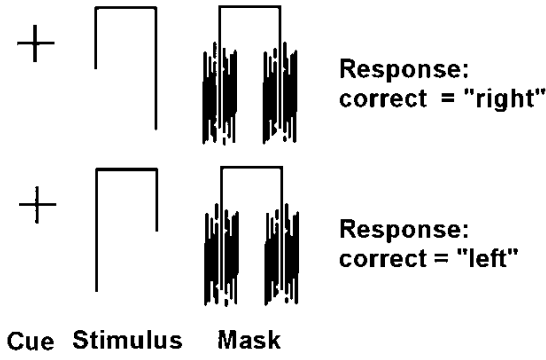

Fig. 2. The cue, stimuli, and backward mask for the inspection time task. See text for procedure.

This is a 60-item test in which subjects examine abstract patterns arrayed as a $3 \times 3$ matrix and, by inducing and then applying the logical rules underlying the rest of the pattern, indicate which of the answer options correctly completes the pattern. The RPM loads highly on the general cognitive ability factor, making it a good indicator of general mental ability [31]. RPM scores obtained in late adulthood and old age correlate (0.7-0.8) significantly with the MHT scores from age 11 [32]. The ABC1936 wave 3 testing data was used to compute the RPM scores of the participant, around age 68 . The ABC1936 participants used in this study had to have taken RPM at late life baseline (age 64) so as to ensure that they had similar experience with the RPM test at age 68. The whole cohort's mean and SD for RPM at age 68 were 37.8 and 7.6 respectively (Table 1).

\section{Brain Imaging Procedures}

MRI data were acquired with a $1.5 \mathrm{~T}$ scanner (NVi, General Electric Medical Systems, Milwaukee, WI, USA) using a standard head coil while performing IT task (160 trials). fMRI data were acquired using a T2* weighted gradient echo echoplanar imaging sequence (EPI) in the axial plane with TR/TE of $2500 / 40 \mathrm{~ms}$, matrix $64 \times 64$, field of view of $24 \mathrm{~cm}^{2}$, thickness of $5 \mathrm{~mm}, 30$ slices per volume. 292 time points/volume in total of which the first 4 volumes were discarded. The total scanning time per fMRI test was $12 \mathrm{~min}$ and $10 \mathrm{~s}$.

\section{Pre-processing}

Spatial pre-processing was performed on the fMRI data using version 5 of Statistical Parametric Mapping (SPM5; The Wellcome Department of Imaging Neuroscience, UCL, London, UK) software. The data were realigned and each voxel time series standardized to a mean of zero and SD of 1.

\section{E. Calculation of ApEn}

ApEn is defined for a given $\mathrm{N}$-dimensional time series $\left(x_{1}, x_{2}, \ldots ., x_{N}\right)$ as:

$$
\begin{aligned}
& \operatorname{ApEn}(m, r, N)=\Phi^{m}(r)-\Phi^{m+1}(r) \\
& \Phi^{m}(r)=[N-(m-1) \tau]^{-1} \sum_{i=1}^{N-(m-1) \tau} \ln C_{i}^{m}(r)
\end{aligned}
$$

Copyright (C) 2011 IEEE. Personal use of this material is permitted. However, permission to use this material for any other purposes must be obtained from the IEEE by sending an email to pubs-permissions@ieee.org 
Where

$$
\begin{gathered}
C_{i}^{m}(r)=\frac{B_{i}}{N-(m-1) \tau} \\
B_{i}=\text { number of } \mathrm{j} \text { where } \mathrm{d}\left|X_{i}, X_{j}\right| \leq r \\
X_{i}=\left(x_{i}, x_{i+\tau}, \ldots ., x_{i+(m-1) \tau}\right) \\
X_{j}=\left(x_{j}, x_{j+\tau}, \ldots ., x_{j+(m-1) \tau}\right) \\
\quad(i, j=1,2, \ldots ., N-(m-1) \tau)
\end{gathered}
$$

In equation (1), $N$ is the number of time points, $m$ specifies the pattern length, $\mathrm{r}$ defines the tolerance value and $\tau$ is the time delay. The two patterns $i$ and $j$ of m measurements of the time series are similar if the difference, $\mathrm{d}\left|X_{i}, X_{j}\right|$ between any pair of corresponding measurements of $X_{i}$ and $X_{j}$ is less than or equal to $\mathrm{r}$, as shown in equation (3). $X_{i}$ and $X_{j}$ are $\mathrm{m}$-dimensional pattern vectors whose components are time delayed versions of the elements in the original time series with time delay, $\tau$ as shown in equation (4) and (5).

ApEn measures the conditional probability of similarity between a chosen data segment of a given duration and the next set of segments of the same duration. When similar patterns in the time series are followed by additional similar patterns, the time series has relatively small ApEn value and when similar patterns are not repetitive it has a more complex pattern.

The parameters used for calculating ApEn are $\mathrm{N}=288$ of fMRI time series, $\mathrm{m}=2, \tau=1$ and $\mathrm{r}=$ the tolerance $(\mathrm{rMx})$ value corresponding to the maximum ApEn (ApEnMx) value multiplied by the Standard Deviation (SD) of the fMRI time series. The choice of $r$ to use has been recommended as $0.1 \leq \mathrm{r} \leq 0.2$ times the SD of the time series [12]. Preliminary analysis of this data indicated that an $\mathrm{r}$ of 0.30 was appropriate (See Appendix A).

The ApEn algorithm was written in MATLAB and measurements on a voxel-by-voxel basis, for the whole brain were implemented. Fig. 3. shows the standardized Blood Oxygen Level Dependent (BOLD) signal of a voxel for one of the volunteers on which the ApEn algorithm was applied. The calculation of ApEn on the whole brain was thresholded at 0.1 times the maximum signal to exclude background voxels. The thresholding was done to prevent voxels outside the brain from being processed and included in the analysis. The mean, median and maximum ApEn values for the whole brain were calculated. Whole brain ApEn maps for each individual were generated. Fig. 4. depicts the ApEn map of a whole brain for one of the volunteers.

\section{F. Statistical Analysis}

Associations between cognitive measures and ApEn were tested on a global and regional basis. The global analysis was

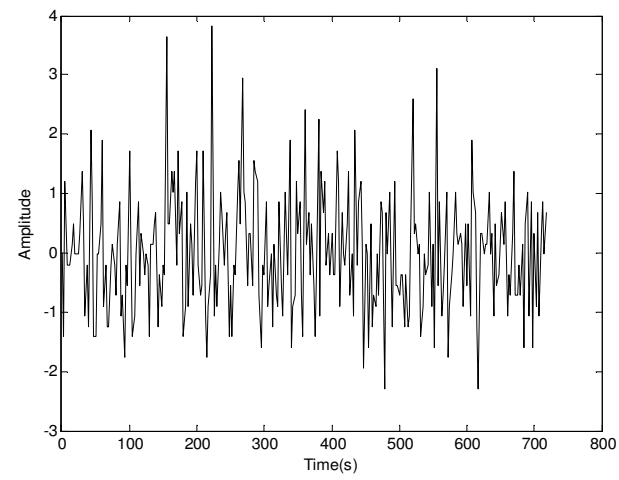

Fig. 3. Standardized BOLD signal for a typical voxel

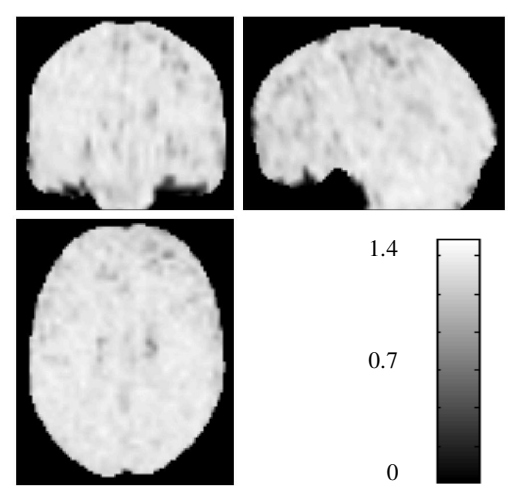

Fig. 4. A 3D whole brain ApEn map of 288 volumes with mean ApEn of 1.1991.

performed using Statistical Package for Social Sciences (SPSS 17.0; Chicago, IL, USA). The ApEn maps of all the participants were normalised to a standard echo planar imaging (EPI) template after which the regional analysis was performed using SPM5. Correlations between the ApEn maps and the IT scores were then found by using standard SPM approach from the second-level group analysis. Correlation coefficients were also calculated between the ApEn maps and IT scores when the MHT and RPM scores were adjusted individually and together.

\section{RESULTS}

Among 58 ABC1936 participants invited for fMRI, 13 fell below a pre-determined threshold of 18 correct responses out of a total of 20 for the $150 \mathrm{~ms}$ duration trials of the IT task. Passing this threshold implied that the participants understood the task and were able to perform it with the easiest stimulus duration. Five of the participants were excluded for medical reasons, such as our inability to correct their vision appropriately. Those who passed the threshold included 40 participants (20 female). Table 1 shows the mean and SD of participant characteristics for the whole ABC1936 sample at wave 3 , the 40 participants who passed the threshold and those who were excluded from the analysis. Examining differences between the excluded participants and those included in the

Copyright (C) 2011 IEEE. Personal use of this material is permitted. However, permission to use this material for any other purposes must be obtained from the IEEE by sending an email to pubs-permissions@ ieee.org 
analysis found no difference between them in terms of their RPM, MHT scores and age at testing. Fig. 5 shows how the participants performed at different trial durations. This was as expected with random responses at the shortest durations and almost perfect responses at the longest durations. The correlations among IT, MHT and RPM are shown in Table 2. The results show only the IT and MHT were not significantly correlated $(\mathrm{p}>0.01)$.

Calculating the mean, median and maximum ApEn values across the whole head, no significant global ApEn associations were found with the IT score obtained from the sum of the number of correct responses at all durations of the IT task. Using an SPM regression approach and an initial threshold of $\mathrm{p}<0.005$ with familywise error (FWE) corrected cluster level significance $(p<0.05 \mathrm{~N}>250)$ [33], Fig. 6(a) shows the regions where there was significant positive correlation between IT and ApEn, i.e. the higher the IT score (better visual information processing) the more complex the fMRI signal, as hypothesized. No negative global or regional associations between IT and ApEn were found during this analysis. Using SPM we went on to examine the regional association between IT and ApEn after adjusting for childhood (MHT) and late life (RPM) cognitive ability. When the association between IT and ApEn was tested after adjusting for MHT, the significant positive correlation of IT with ApEn remained with some minimal changes in brain regions. After adjusting for RPM, the significant positive correlation of ApEn with IT remained, but with significant changes in brain regions. When both age 11 IQ (MHT) and RPM were adjusted for, there was no correlation between IT and ApEn. Also, when the standardized difference between age 11 IQ (MHT) and RPM (life long cognitive change) was adjusted for, there was no correlation between IT and ApEn. Table 3 shows the association of IT with ApEn and adjustment for MHT and RPM separately.

These results suggest that the ApEn-IT association was explained by life long cognitive change. Examining this suggestion closer, investigating effect size is difficult in terms of the cluster level significances we have demonstrated since they are a combination of contrast ( $\mathrm{t}$ or $\mathrm{Z}$ statistic) and extent (size of the cluster). However, extracting peak $\mathrm{t}$ value in each cluster showed that adjusting for age 11 IQ (MHT) had a small

Table 1: Participants characteristics. Mean (SD) for each group.

\begin{tabular}{llll}
\hline & $\begin{array}{l}\text { The ABC1936 } \\
\text { sample at age } \\
\mathbf{6 8}(\mathbf{N = 3 1 3})\end{array}$ & $\begin{array}{l}\text { The 40 participants } \\
\text { who passed the } \\
\text { threshold }\end{array}$ & $\begin{array}{l}\text { The 13 } \\
\text { excluded } \\
\text { participants }\end{array}$ \\
\hline $\begin{array}{l}\text { Moray House } \\
\text { Test score at } \\
\text { age } 11^{*}\end{array}$ & $103.7(13.1)$ & $105.7(6.7)$ & $100.0(12.5)$ \\
\hline $\begin{array}{l}\text { Raven's } \\
\text { Progressive } \\
\text { matrices }\end{array}$ & $37.8(7.6)$ & $39.8(9.2)$ & $39.5(6.8)$ \\
\hline $\begin{array}{l}\text { Total number } \\
\text { of correct } \\
\text { responses (out } \\
\text { of 160) }\end{array}$ & - & $111.5(9.3)$ & - \\
\hline $\begin{array}{l}\text { Age at testing } \\
\text { years) }\end{array}$ & $68.8(0.7)$ & & \\
\hline$* \quad$ The Moray & House Test scores were standardized using all of the \\
participants recruited at age 64 years $(\mathrm{N}=661)$ &
\end{tabular}

effect decreasing the $r^{2}$ estimate between IT and ApEn. RPM had a moderate effect decreasing the $r^{2}$ value between IT and ApEn and adjusting for life long cognitive change had a large effect decreasing the $r^{2}$ estimate between IT and ApEn. For example using cluster D (Table 3 ), the cluster of median size and the peak voxel located at $38,0,-14$ an $r^{2}=.257$ value was calculated for the ApEn-IT model. Adjusting this association for age 11 IQ (MHT) the $\mathrm{r}^{2}$ was .242 (6\% effect). Adjusting for current ability (RPM) the $\mathrm{r}^{2}$ was .147 (43\% effect). Adjusting for life long cognitive change (standardized difference between MHT and RPM) the $\mathrm{r}^{2}$ was $.014(95 \%$ effect). The dotted lines in Fig. 1 show how our original schematic structure is informed by our results. We found no evidence for an influence of childhood ability on IT and ApEn.

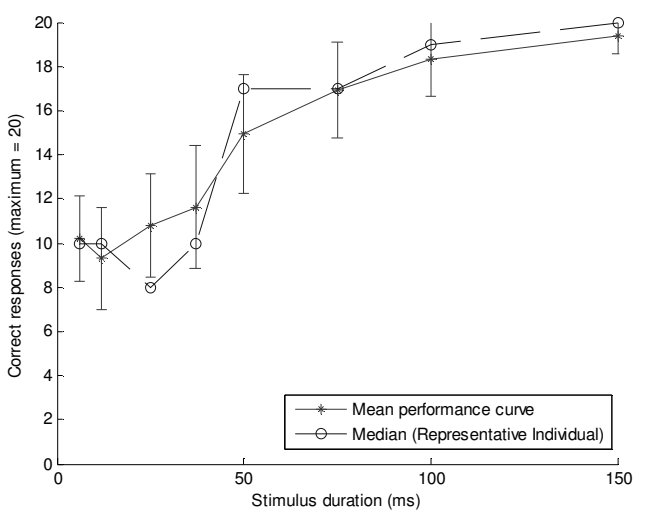

Fig. 5. The group mean performance curve on the IT task (mean \pm 2 SD) and median (Representative Individual).

Table 2: Correlations among IT, MHT (age 11) and RPM (age 68).

\begin{tabular}{llll}
\hline & $\begin{array}{l}\text { IT - MHT } \\
\text { correlation } \\
(\mathbf{N}=\mathbf{4} \text { 0) }\end{array}$ & $\begin{array}{l}\text { IT - RPM } \\
\text { correlation } \\
\mathbf{( N = 4 0 )}\end{array}$ & $\begin{array}{l}\text { MHT - RPM } \\
\text { correlation } \\
\mathbf{N = 4 0 )}\end{array}$ \\
\hline $\begin{array}{l}\text { Pearson } \\
\text { Correlation* }\end{array}$ & 0.213 & 0.466 & 0.489 \\
\hline $\begin{array}{l}\text { Significance } \\
\text { (2-tailed) }\end{array}$ & 0.188 & 0.002 & 0.001 \\
\hline
\end{tabular}

* Correlation is significant at the 0.01 level (2-tailed).

\section{DISCUSSION}

These results show that high ApEn values at some grey and white matter locations throughout the brain, while performing the IT task, are associated with better cognitive performance. This is consistent with our hypothesis that higher levels of entropy will be associated with better cognitive performance. These regions have previously been shown to be associated with visual processing tasks and speed, using structural and functional imaging. The cerebellum has previously been found to be involved in age related differences in visual processing speed [34] and has been found to contribute to general cognitive ability independent of other brain structures [35]. Neurophysiological and neuropsychological evidence have implicated temporal regions in visual discrimination and inferior parietal regions in the allocation of attention to locations in the visual field [36]. The frontal gyrus is

Copyright (C 2011 IEEE. Personal use of this material is permitted. However, permission to use this material for any other purposes must be obtained from the IEEE by sending an email to pubs-permissions@ ieee.org 
associated with high-level executive functions and decisionrelated processes [37]. The insula is associated with visual anticipation which is a critical component for visual processing [38]. The basal ganglia have been associated with cognitive fatigue with time [39]. The posterior cingulate has been shown to be involved in Alzheimer's disease and associated with reduced processing speed in older adults using resting state fMRI [40]. Collectively, the literature shows that each of the locations identified using entropy has previously been associated with abilities that intuitively would contribute to superior information processing ability as we have measured it.

The locations found (as a result of a significant positive correlation between IT performance and ApEn) in this study have some similarities with those found in this data set by Waiter et al. [28] using a conventional fMRI analysis approach investigating BOLD response correlations with IT duration.

The study found that the relative preservation of cognition in old age may be associated with the preservation of fundamental information processing networks found in the young. A location in the posterior cingulate and left precuneus is common to both analyses, shown in Fig. 6(b). Waiter et al. also found a negative association between activation and IT in the posterior cingulate and the left precuneus. This suggests that the variety of signal patterns produced while performing the IT task is an indication of ability. A greater variety of signal pattern will give larger entropy. Our analysis also found associations in a number of regions, described above which were not identified by the previous analysis. These regions do not exhibit a measureable response (measured using contrast) during the task and may be part of an extended network used to perform it. Estimates of signal entropy at these locations may, therefore, be a proxy for ability, or conversely decreasing entropy in these regions may be an early correlate of cognitive decline. IT and intelligence have a well-established significant association of moderate effect size, and so any association with entropy may be due to intelligence rather than being specific to the task. The survival of the ApEn-IT associations after adjusting for concurrent intelligence (RPM) suggests that it is the non-intelligence variance in IT that is in part responsible for association at these locations.

The minor impact on the regional results after adjusting for MHT indicates that early life ability does not explain the ApEn-IT association. Similarly, the considerable impact of adjusting for RPM on the regional result indicates that at least part of the ApEn-IT association is explained by current higher cognitive abilities. Since MHT and RPM are significantly correlated it could be that it is the variance in RPM not explained by MHT that explains the ApEn-IT association. After adjustment for the standardized difference between MHT and RPM (life long cognitive change) no significant ApEn-IT association was observed. The adjustment resulted in an $\mathrm{r}^{2}$ reduction of $95 \%$ in the ApEn-IT association at particular locations. In other words, higher cognitive abilities acquired since age 11 explain the ApEn-IT association. These changes may be brought about by life experiences such as educational and occupational attainment. More education and a more cognitively complex occupation predict higher cognitive ability in old age than would be expected for a person's childhood ability and accumulated brain burden [41] who found that the intellectual challenges experienced during life, such as education and occupation, accumulate reserve and allow cognitive function to be maintained in old age. In addition to these mental or intellectual activities, genetics, lifestyle and diet have also been shown to maintain ability in late life [25]. It is clear that the factors affecting the ability of an individual to adapt to the changing demands of ageing and disease are multifactor in nature.

This study departs from previously recommended tolerance values, $\mathrm{r}$ of $0.1 \leq \mathrm{r} \leq 0.2$ times the SD [12]. The tolerance value we have used corresponds to the ApEnMx value. This was calculated as part of our initial analysis of the data to be 0.3 (Appendix A). The choice of $r$ is possibly data set specific. Here we have selected $r$ based on the ApEn variance it produced between individuals and minimising the sensitivity of ApEn to r. It was clear from our initial analysis that an individual's ApEnMx occurred at slightly different values of $r$. We would recommend investigating this relationship prior to examining individual differences between subjects as inappropriate selection could lead to reduced sensitivity and/or erroneous findings.

The limitation of fMRI and other functional approaches such as functional positron emission tomography, magnetoencephalography and electroencephalogram are that they predominantly rely on a stimulus initiated response for signals to be detected and locations and or network to be implicated. All parts of the brain involved in a particular function are unlikely to demonstrate a measurable stimulus induced response. Measuring signal entropy in this way may identify locations and networks that are required for functions that do not demonstrate this stimulus induced response. This study has been conducted in a narrow age range which restricts generalizing our findings to other age ranges, particularly younger ones. Our previous work using these data [28] has noted that successful ageing produced activation patterns similar to that found in a younger sample [27] for this task. It is unclear if measures of entropy would be similar in the younger samples that were without age related brain changes. In addition in this study we have selected participants based on a limited range of childhood abilities. This could have restricted the ability of the MHT to explain the association between ApEn and IT and it is possible that including those with a wider range of childhood ability may reveal an association with ApEn.

A limitation of this study includes the fact that we had a short time series. ApEn is dependent on time series length. A superior estimation of ApEn may be obtained from longer time series since a longer time series corresponds to higher ApEn values which gives a better approximation of the entropy. Our study was task dependent; therefore the variability of entropy using the same, different and no task requires further investigation. Alternative measures of complexity are Sample Entropy (SampEn), Multiscale ApEn, Multiscale SampEn and Fractal analysis which have found use in a variety of applications. However, the optimal approach for fMRI data such as this is unclear. 

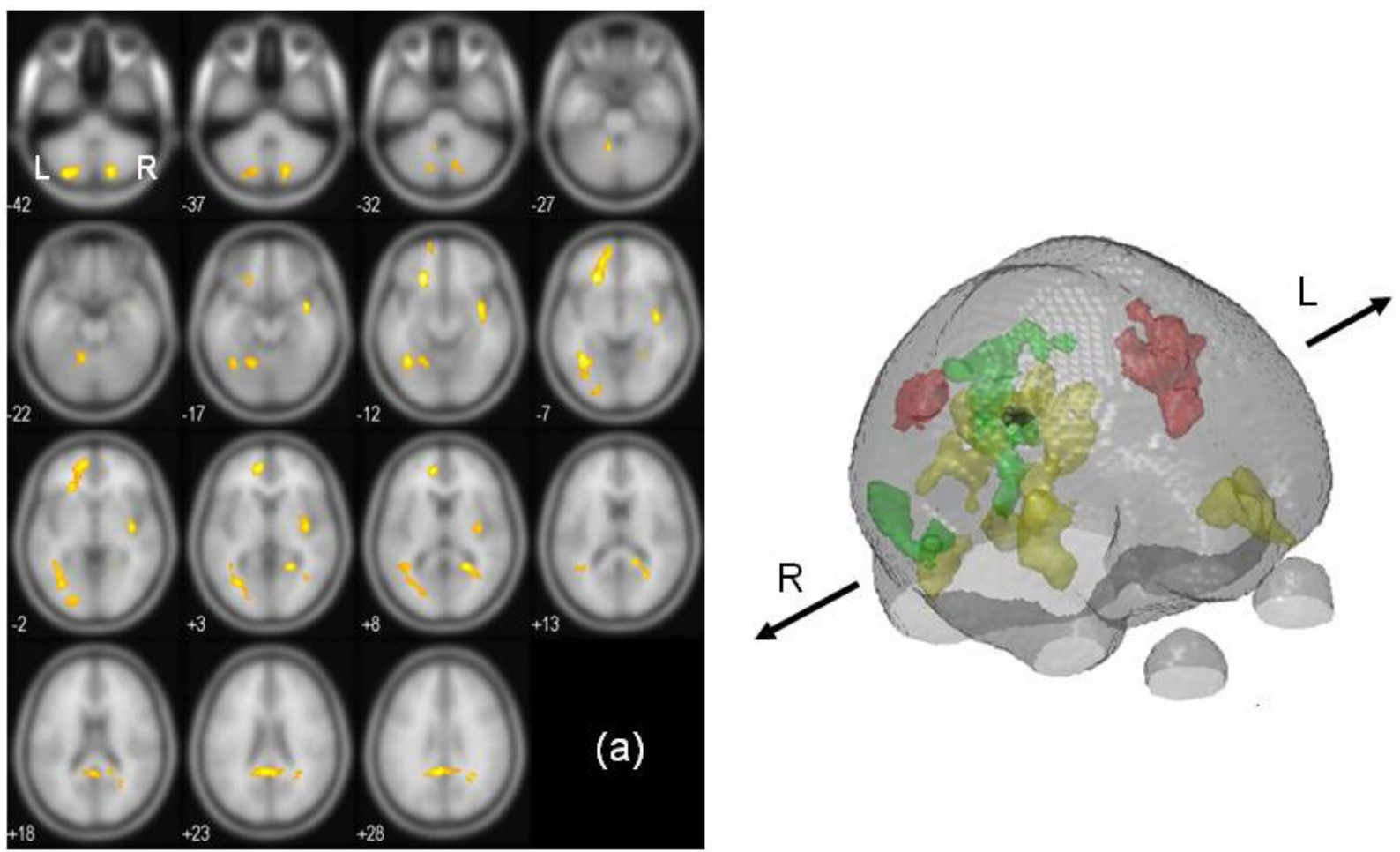

Fig. 6. (a) Axial image slices of regions in the brain showing significant positive correlations between IT and ApEn. (b) A 3D image showing a comparison between the conventional fMRI analysis approach (green/red) of this data (Waiter et al. [32]) at a threshold of $(\mathrm{p}<0.005, \mathrm{~N}>250$, corrected cluster $\mathrm{p}<0.05)$ and the ApEn-IT findings (yellow). Yellow represents the significant ApEn-IT correlations, green represents negative activations during the IT task, red represents the positive activation during the IT task. The overlap (black) is located at Left Cerebrum, Limbic Lobe, Posterior Cingulate, Left Precuneus.

\section{CONCLUSION}

To the best of our knowledge, this is the first use of temporal signal entropy to investigate individual differences in fMRI related to late life cognitive ability. Our results show that individual differences in IT are associated with regional differences in ApEn calculated from fMRI data. These results suggest that the association between IT and ApEn is a consequence of life long cognitive change, rather than early life ability. Explicitly, these results suggest that differences in life long cognitive change explains patterns of brain activity measured using fMRI and entropy. These changes are brought about by the balance between subclinical pathology, genetic predisposition and environment. In addition we have shown that a measure of fMRI temporal signal entropy is a viable tool to identify a potential source of individual differences.

\section{APPENDIX A}

Estimation of Tolerance value, $r$ in the Calculation of ApEn

The tolerance value, $r$ is essentially a filter, where the type of filter depends on the choice of $r$. The choice of $r$ to use has been recommended as $0.1 \leq \mathrm{r} \leq 0.2$ times the $\mathrm{SD}$ of the time series [12]. This range of $r$ has been used with applications with relatively slow dynamic signals such as heart rate variation [42],[43] and hormonal release data [44]. Recent studies have shown that this recommended range is not always appropriate for fast dynamic neural signals [45]. FMRI data are an example of signals that are the result of fast dynamic neural signals. In order to compare like with like, a fixed value of $r$ was required. An appropriate value of $r$ is one that produces a large enough variance in ApEn, between individuals to detect differences. The value of $r$ should be such that minor changes in its value do not produce large changes in ApEn. In addition, the selection of $r$ should be based on a reproducible strategy. For example here we have selected a value of $r$ that gives the largest value of ApEn. Fig. A. shows the plot of ApEn versus $r$ for two of the volunteers, $\mathrm{S} 1$ and $\mathrm{S} 2$. Here, the rMx value for $\mathrm{S} 1$ and $\mathrm{S} 2$ are 0.28 and 0.32 respectively. The rMx values occur in a flat region of the curve. The recommended values of $r$ are in the steepest part of the curve where a small variation in $r$ would produce a large change in ApEn. A plot of ApEn versus $r$ for 30 of the volunteers using the algorithm described in the methods 
Table 3: Location that show a positive association of IT with ApEn before and after adjustment for MHT and RPM (with initial threshold of $\mathrm{p}<0.005$ and extent voxel of $\mathrm{N}>250$ ), ns - not significant.

\begin{tabular}{|c|c|c|c|c|c|c|c|c|}
\hline $\begin{array}{l}\text { Cluster } \\
\text { ID }\end{array}$ & $\begin{array}{l}\text { Talairach } \\
\text { coordinate } \\
(\mathrm{XYZ})\end{array}$ & $\begin{array}{l}\text { Regions in the brain included in each } \\
\text { cluster }\end{array}$ & $\begin{array}{c}\text { Cluster p } \\
\text { value (FWE } \\
\text { corrected) } \\
\text { (ApEn-IT } \\
\text { correlation) }\end{array}$ & $\begin{array}{l}\text { Cluster } \\
\text { Extent } \\
\text { (ApEn-IT } \\
\text { correlation) }\end{array}$ & $\begin{array}{c}\text { Cluster p } \\
\text { value } \\
\text { (FWE } \\
\text { corrected) } \\
\text { (ApEn-IT } \\
\text { correlation } \\
\text { after } \\
\text { adjusting } \\
\text { for MHT) }\end{array}$ & $\begin{array}{c}\text { Cluster } \\
\text { Extent } \\
\text { (ApEn-IT } \\
\text { correlation } \\
\text { after } \\
\text { adjusting } \\
\text { for MHT) } \\
\end{array}$ & $\begin{array}{c}\text { Cluster p } \\
\text { value } \\
\text { (FWE } \\
\text { corrected) } \\
\text { (ApEn-IT } \\
\text { correlation } \\
\text { after } \\
\text { adjusting } \\
\text { for RPM) }\end{array}$ & $\begin{array}{c}\text { Cluster } \\
\text { Extent } \\
\text { (ApEn-IT } \\
\text { correlation } \\
\text { after } \\
\text { adjusting } \\
\text { for RPM) } \\
\end{array}$ \\
\hline A & $-26-76-44$ & $\begin{array}{l}\text { Left Cerebellum, Cerebellum Posterior } \\
\text { Lobe, Pyramis }\end{array}$ & 0.000 & 360 & 0.000 & 417 & ns & - \\
\hline B & $16-76-40$ & $\begin{array}{l}\text { Right Cerebellum, Cerebellum Posterior } \\
\text { Lobe, Pyramis }\end{array}$ & 0.002 & 293 & 0.001 & 325 & ns & - \\
\hline $\mathrm{C}$ & $\begin{array}{llll}-38 & -54 & -8 \\
-32 & -68 & 2 \\
-10 & -52 & -26\end{array}$ & $\begin{array}{l}\text { Left Cerebrum, Temporal Lobe, Sub- } \\
\text { Gyral, White Matter } \\
\text { Left Cerebrum, Occipital Lobe, Middle } \\
\text { Occipital Gyrus, White Matter } \\
\text { Left Cerebellum, Anterior Lobe, Gray } \\
\text { Matter, Dentate }\end{array}$ & 0.000 & 1214 & 0.000 & 1737 & 0.000 & 941 \\
\hline D & $\begin{array}{lll}38 & 0 & -14 \\
38 & -10 & -6 \\
28 & 0 & 6\end{array}$ & $\begin{array}{l}\text { Right Cerebrum, Temporal Lobe, } \\
\text { Superior Temporal Gyrus, White Matter } \\
\text { Right Cerebrum, Sub-lobar, Insula, } \\
\text { White Matter } \\
\text { Right Cerebrum, Sub-lobar, Lentiform } \\
\text { Nucleus, Gray Matter, Putamen }\end{array}$ & 0.000 & 436 & ns & - & ns & - \\
\hline $\mathrm{E}$ & $\begin{array}{lll}-22 & 30 & -10 \\
-12 & 48 & 6 \\
-16 & 50 & -4\end{array}$ & $\begin{array}{l}\text { Left Cerebrum, Frontal Lobe, Inferior } \\
\text { Frontal Gyrus, Gray Matter } \\
\text { Left Cerebrum, Frontal Lobe, Medial } \\
\text { Frontal Gyrus, White Matter } \\
\text { Left Cerebrum, Frontal Lobe, Medial } \\
\text { Frontal Gyrus, White Matter }\end{array}$ & 0.000 & 757 & 0.000 & 659 & 0.000 & 793 \\
\hline $\mathrm{F}$ & $\begin{array}{lll}24 & -52 & 8 \\
30 & -52 & 32 \\
16 & -46 & 14\end{array}$ & $\begin{array}{l}\text { Right Cerebrum, Sub-lobar, Extra- } \\
\text { Nuclear, White Matter } \\
\text { Right Cerebrum, Parietal Lobe, Sub- } \\
\text { Gyral, White Matter } \\
\text { Right Cerebrum, Sub-lobar, Extra- } \\
\text { Nuclear, White Matter, Corpus Callosum }\end{array}$ & 0.000 & 437 & 0.000 & 394 & ns & - \\
\hline G & $\begin{array}{lll}-4 & -46 & 26 \\
4 & -48 & 24\end{array}$ & $\begin{array}{l}\text { Left Cerebrum, Limbic Lobe, Cingulate } \\
\text { Gyrus, White Matter } \\
\text { Right Cerebrum, Limbic Lobe, Posterior } \\
\text { Cingulate, Gray Matter }\end{array}$ & 0.000 & 370 & 0.001 & 351 & ns & - \\
\hline
\end{tabular}

section was produced. The mean ApEn of 100 voxels in the frontal lobe, sub-lobar and occipital lobe of the brain for each of these volunteers was evaluated with $\mathrm{r}$ increasing from 0 to 0.6 at intervals of 0.02 . The rMx value corresponding to the ApEnMx value in the frontal lobe, sub-lobar and occipital lobe of the brain of each of the 30 volunteers was obtained and the median rMx value was calculated as 0.3 . This median $\mathrm{rMx}$ value was used as the value of $r$ in the main study.

\section{ACKNOWLEDGMENT}

The authors would like to thank J. Roijals-Miras who performed some initial analysis on this data. They also thank the people of Aberdeen for giving their time and effort when helping with the study, to local family doctors for providing access to their patients and to members of the research team not listed as authors. These were: Jen Herbert, Steve Leaper, Duncan Leitch, Mariesha Struth, Linda Taylor, Tor Bourne and Charlotte Appleton.

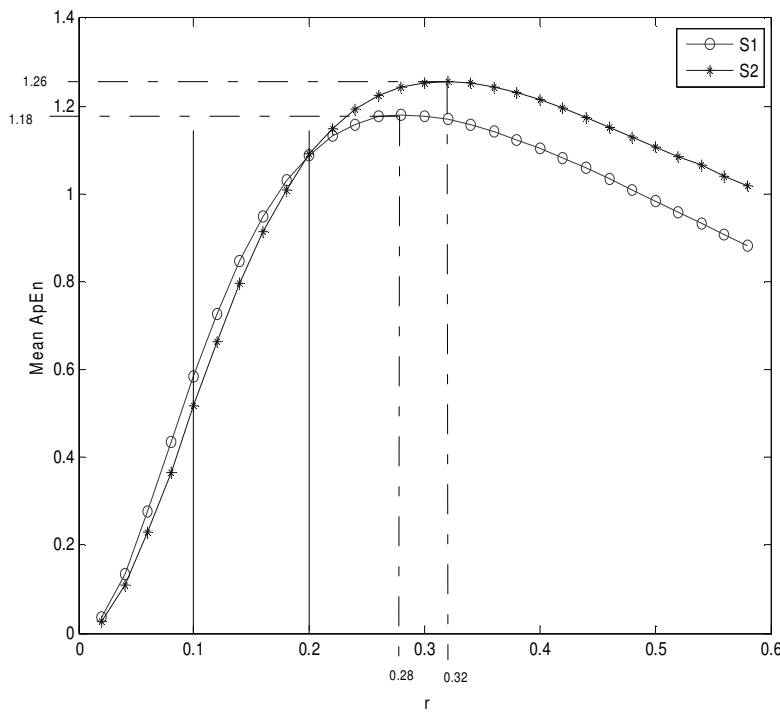

Fig. A. Plot of ApEn versus $r$ at the sub-lobar of the brain for two volunteers. The two solid vertical lines on 0.1 and 0.2 delimitate the recommended $r$ range.

Copyright (C) 2011 IEEE. Personal use of this material is permitted. However, permission to use this material for any other purposes must be obtained from the IEEE by sending an email to pubs-permissions@ @ieee.org 


\section{REFERENCES}

[1] C. Brayne, P. G. Ince, H. A. D. Keage, I. G. McKeith, F. E. Matthews, T. Polvikoski and R. Sulkava, "Education, the brain and dementia: Neuroprotection or compensation?" Brain, vol. 133, pp. 2210-2216, 2010 [2] Y. Stern, "Cognitive reserve," Neuropsychologia, vol. 47, pp. 2015-2028, 2009.

[3] M. Richards and I. J. Deary, "A life course approach to cognitive reserve: A model for cognitive aging and development?" Ann. Neurol., vol. 58, pp. 617-622, 2005

[4] L. J. Whalley, I. J. Deary, C. L. Appleton and J. M. Starr, "Cognitive reserve and the neurobiology of cognitive aging," Ageing Res. Rev., vol. 3, pp. 369-382, 2004.

[5] L. A. Lipsitz, "Dynamics of stability: The physiologic basis of functiona health and frailty," J. Gerontol. Ser. A Biol. Sci. Med. Sci., vol. 57, 2002. [6] L. A. Lipsitz, "Physiological complexity, aging, and the path to frailty." Science of Aging Knowledge Environment [Electronic Resource] : SAGE $K E$, vol. 2004, 2004.

[7] A. L. Goldberger, C. -. Peng and L. A. Lipsitz, "What is physiologic complexity and how does it change with aging and disease?" Neurobiol. Aging, vol. 23, pp. 23-26, 2002.

[8] D. T. Kaplan, M. I. Furman, S. M. Pincus, S. M. Ryan, L. A. Lipsitz and A. L. Goldberger, "Aging and the complexity of cardiovascular dynamics," Biophys. J., vol. 59, pp. 945-949, 1991.

[9] R. Pool, "Is it healthy to be chaotic?" Science, vol. 243, pp. 604-607, 1989.

[10] A. J. Mandell and M. F. Schlesinger, "Lost choices: Parallelism and topological entropy decrements in neurobiological aging," The Ubiquity of Chaos, pp. 35-46, 1990.

[11] L. Balducci, "Aging, frailty, and chemotherapy," Cancer Control, vol. 14, pp. 7-12, 2007.

[12] S. M. Pincus, "Approximate entropy as a measure of system complexity," Proc. Natl. Acad. Sci. U. S. A., vol. 88, pp. 2297-2301, 1991.

[13] S. M. Pincus, "Assessing serial irregularity and its implications for health," Ann. New York Acad. Sci., vol. 954, pp. 245-267, 2001.

[14] S. M. Pincus and D. L. Keefe, "Quantification of hormone pulsatility via an approximate entropy algorithm," American Journal of Physiology Endocrinology and Metabolism, vol. 262, 1992

[15] S. M. Ryan, A. L. Goldberger, S. M. Pincus, J. Mietus and L. A. Lipsitz, "Gender- and age-related differences in heart rate dynamics: Are women more complex than men?" J. Am. Coll. Cardiol., vol. 24, pp. 1700-1707, 1994. [16] S. A. Caswell Schuckers and P. Raphisak, "Distinction of arrhythmias with the use of approximate entropy," in 1999, pp. 347-350.

[17] N. Burioka, G. Cornélissen, F. Halberg, D. T. Kaplan, H. Suyama, T. Sako and E. Shimizu, "Approximate entropy of human respiratory movement during eye-closed waking and different sleep stages," Chest, vol. 123, pp. 8086,2003

[18] W. J. Levy, E. Pantin, S. Mehta and M. McGarvey, "Hypothermia and the approximate entropy of the electroencephalogram," Anesthesiology, vol. 98, pp. 53-57, 2003.

[19] D. Abasolo, R. Hornero, P. Espino, A. Alonso and R. de la Rosa "Electroencephalogram analysis with approximate entropy to help in the diagnosis of Alzheimer's disease," Proceedings of the 4th International IEEE EMBS Special Topic Conference on Information Technology Applications in Biomedicine, pp. 222-225, 2004.

[20] T. A. Braver and R. West, Eds., Working Memory, Executive Control, and Aging, in: Craik, F.I.M., Salthouse, T.A. (Eds.), the Handbook of Cognition and Aging. New York: Psychology Press, 2008.

[21] D. Finkel, C. A. Reynolds, J. J. McArdle and N. L. Pedersen, "The longitudinal relationship between processing speed and cognitive ability: Genetic and environmental influences," Behav. Genet., vol. 35, pp. 535-549, 2005.

[22] T. A. Salthouse, "The Processing-Speed Theory of Adult Age Differences in Cognition," Psychol. Rev., vol. 103, pp. 403-428, 1996. [23] I. J. Deary, "Human intelligence differences: Towards a combined experimental-differential approach," Trends Cogn. Sci., vol. 5, pp. 164-170, 2001.

[24] I. J. Deary, L. J. Whalley, H. Lemmon, J. R. Crawford and J. M. Starr, "The stability of individual differences in mental ability from childhood to old age: Follow-up of the 1932 Scottish mental survey," Intelligence, vol. 28, pp. 49-55, 2000.
[25] I. J. Deary, J. Corley, A. J. Gow, S. E. Harris, L. M. Houlihan, R. E. Marioni, L. Penke, S. B. Rafnsson and J. M. Starr, "Age-associated cognitive decline," Br. Med. Bull., vol. 92, pp. 135-152, 2009.

[26] Scottish Council for Research in Education, "The trend of scottish intelligence," University of London Press, London, 1949.

[27] I. J. Deary, E. Simonotto, M. Meyer, A. Marshall, I. Marshall, N. Goddard and J. M. Wardlaw, "The functional anatomy of inspection time: An event-related fMRI study," Neuroimage, vol. 22, pp. 1466-1479, 2004. [28] G. D. Waiter, H. C. Fox, A. D. Murray, J. M. Starr, R. T. Staff, V. J. Bourne, L. J. Whalley and I. J. Deary, "Is retaining the youthful functional anatomy underlying speed of information processing a signature of successful cognitive ageing? An event-related fMRI study of inspection time performance," Neuroimage, vol. 41, pp. 581-595, 2008.

[29] G. D. Waiter, I. J. Deary, R. T. Staff, A. D. Murray, H. C. Fox, J. M Starr and L. J. Whalley, "Exploring possible neural mechanisms of intelligence differences using processing speed and working memory tasks: An fMRI study," Intelligence, vol. 37, pp. 199-206, 2009.

[30] J. C. Raven, J. H. Court and J. Raven, "Manual for raven's progressive matrices and vocabulary scales," H.K. Lewis, London, 1977.

[31] J. B. Carroll, Ed., Human Cognitive Abilities: A Survey of Factor Analytic Studies. Cambridge: Cambridge University Press, 1993.

[32] I. J. Deary, M. C. Whiteman, J. M. Starr, L. J. Whalley and H. C. Fox, "The Impact of Childhood Intelligence on Later Life: Following Up the Scottish Mental Surveys of 1932 and 1947," J. Pers. Soc. Psychol., vol. 86, pp. 130-147, 2004

[33] T. Nichols and S. Hayasaka, "Controlling the familywise error rate in functional neuroimaging: A comparative review," Stat. Methods Med. Res., vol. 12, pp. 419-446, 2003.

[34] M. A. Eckert, N. I. Keren, D. R. Roberts, V. D. Calhoun and K. C. Harris, "Age-related changes in processing speed: Unique contributions of cerebellar and prefrontal cortex," Front. Human Neurosci., vol. 4, 2010.

[35] M. J. Hogan, R. T. Staff, B. P. Bunting, A. D. Murray, T. S. Ahearn, I. J. Deary and L. J. Whalley, "Cerebellar brain volume accounts for variance in cognitive performance in older adults," Cortex, vol. 47, pp. 441-450, 2011. [36] L. C. Robertson and M. R. Lamb, "Can deficits in attention account for global local dissociations in brain-damaged patients," Journal of Clinical and Experimental Neuropsychology, vol. 10, pp. 28-28, 1988.

[37] A. Talati and J. Hirsch, "Functional specialization within the medial frontal gyrus for perceptual go/no-go decisions based on "what," "when," and "where" related information: An fMRI study," J. Cogn. Neurosci., vol. 17, pp. 981-993, 2005.

[38] A. Simmons, I. Strigo, S. C. Matthews, M. P. Paulus and M. B. Stein, "Anticipation of Aversive Visual Stimuli Is Associated With Increased Insula Activation in Anxiety-Prone Subjects," Biol. Psychiatry, vol. 60, pp. 402409, 2006.

[39] J. DeLuca, H. M. Genova, F. G. Hillary and G. Wylie, "Neural correlates of cognitive fatigue in multiple sclerosis using functional MRI," J. Neurol. Sci., vol. 270, pp. 28-39, 2008

[40] J. S. Damoiseaux, C. F. Beckmann, E. J. S. Arigita, F. Barkhof, P. Scheltens, C. J. Stam, S. M. Smith and S. A. R. B. Rombouts, "Reduced resting-state brain activity in the "default network" in normal aging," Cereb. Cortex, vol. 18, pp. 1856-1864, 2008.

[41] R. T. Staff, A. D. Murray, I. J. Deary and L. J. Whalley, "What provides cerebral reserve?" Brain, vol. 127, pp. 1191-1199, 2004.

[42] G. S. Dawes, M. Moulden, O. Sheil and C. W. G. Redman,

"Approximate entropy, a statistic of regularity, applied to fetal heart rate data before and during labor," Obstet. Gynecol., vol. 80, pp. 763-768, 1992.

[43] S. M. Pincus and R. R. Viscarello, "Approximate entropy: A regularity measure for fetal heart rate analysis," Obstet. Gynecol., vol. 79, pp. 249-255, 1992.

[44] X. Chen, K. H. Chon and I. C. Solomon, "Chemical activation of preBötzinger complex in vivo reduces respiratory network complexity," American Journal of Physiology - Regulatory Integrative and Comparative Physiology, vol. 288, 2005.

[45] S. Lu, X. Chen, J. K. Kanters, I. C. Solomon and K. H. Chon,

"Automatic selection of the threshold value $\mathrm{r}$ for approximate entropy," IEEE Transactions on Biomedical Engineering, vol. 55, pp. 1966-1972, 2008.

Copyright (C 2011 IEEE. Personal use of this material is permitted. However, permission to use this material for any other purposes must be obtained from the IEEE by sending an email to pubs-permissions@ ieee.org 